\title{
Completion Pneumonectomy
}

National Cancer Institute

\section{Source}

National Cancer Institute. Completion Pneumonectomy. NCI Thesaurus. Code C91831.

A surgical procedure involving the removal of the entire remaining lung tissue following partial lung resection. 